

\title{
O IMPACTO DO CRM SOCIAL EM PEQUENAS EMPRESAS INSERIDAS EM AMBIENTES B2B
}

\author{
THE IMPACT OF SOCIAL CRM ON B2B AND SMES
}

\section{iD Charles Ristow ${ }^{1}$ \\ (iD) Edna Gessner ${ }^{2}$ \\ DEdson Pacheco Paladini ${ }^{3}$}

\author{
${ }^{1}$ Mestre \\ Universidade Federal de Santa Catarina - UFSC. \\ Florianópolis, Santa Catarina - Brasil. \\ charles.ristow@gmail.com

\section{${ }^{2}$ Mestre} \\ Universidade Federal de Santa Catarina - UFSC. \\ Florianópolis, Santa Catarina - Brasil. \\ edna.gessner@gmail.com \\ ${ }^{3}$ Prof. Doutor \\ Universidade Federal de Santa Catarina - UFSC. \\ Florianópolis, Santa Catarina - Brasil. \\ paladini@floripa.com.br
}

Recebido em: 21 ago. 2019

Aprovado em: 13 fev. 2020
Resumo: O presente estudo aborda a qualidade do relacionamento com o cliente no contexto business-to-business (B2B) e avalia a utilização de sistemas do tipo Customer Relationship Management (CRM) baseado na utilização de tecnologias Web 2.0 e mídias sociais. A empresa objeto do estudo é uma cervejaria que utiliza um sistema do tipo CRM social para aumento da confiança no processo de comunicação e da sua capacidade de resposta, além da satisfação do cliente em termos de atendimento individualizado. Adicionalmente propôs-se a utilização de indicadores para avaliar o processo, que consideraram a performance em vendas, nível de atendimento, escala de utilização e efetividade na utilização das mídias sociais. O estudo evidencia que a adoção de sistemas do tipo CRM Social é importante para a empresa do estudo, devido ao interesse na construção de relacionamentos de longo prazo, bem como para a prospeç̧ão de novos clientes.

Palavras-chave: CRM social. Qualidade no relacionamento. Cervejaria artesanal.

Abstract: The present study addresses the quality of the customer relationship in the business-to-business (B2B) context and the use of customer relationship management (CRM) systems using Web 2.0 technologies and social media. The case study was conducted in a microbrewery. The company adopted a CRM system to increase the level of trust in the communication process and responsiveness, as well as customer satisfaction in terms of individualized service. Some KPIs are proposed to evaluate the process, sales performance, service level and effectiveness in the use of social media. In practice, the study shows that CRM data management systems aim to build long-term relationships and prospecting new customers.

Keywords: Social CRM. Relationship quality. Craft brewing. 
1 Introdução

Para sobreviver em um mercado cada vez mais competitivo, as empresas precisam se diferenciar. Tal diferenciação não se restringe apenas a promoções ou produtos inovadores. Para atrair compras futuras, é necessário que a empresa conheça e mantenha boas relações com seus clientes, a fim de atender às necessidades individuais dos mesmos. No contexto business-to-business (B2B), ou seja, entre empresas, a importância da qualidade do relacionamento é amplamente reconhecida, e está relacionada à força e continuidade dos relacionamentos inter-firmas e sua influência no desempenho das operações das empresas.

A implementação de estratégias de gestão de relacionamento, a exemplo do Customer Relationship Management (CRM), visa à obtenção de dados e informações aprimoradas do cliente, que não se originam apenas do volume de vendas (Pedron et al., 2016; Soltani \& Navimipour, 2016). O CRM une o potencial da tecnologia de informação às estratégias para qualidade do relacionamento entre empresas, com o cliente e parceiros comerciais. Atualmente, a tendência e o desafio nesses sistemas são a combinação de dados de mídias sociais ao CRM, visto que a utilização dessas novas tecnologias vem amadurecendo e essa integração pode fornecer as informações necessárias para desenvolver soluções sob medida à demanda dos clientes (Agnihotri et al., 2016; Cruz-Jesus et al., 2019; Guha et al., 2018).

Pequenas e médias empresas (PMEs), que representam parcela importante na economia de países em desenvolvimento como o Brasil, e que diferem de grandes empresas especialmente em termos de recursos disponíveis, têm atraído a atenção de desenvolvedores de CRM. As PMEs são empresas que se beneficiam de atuação em redes de empresas, colaboração e outras parcerias no contexto B2B, visto que, ao compartilhar recursos, informações e/ou conhecimento, as restrições devido ao porte das mesmas podem ser superadas. Assim, sistemas relacionados à qualidade do relacionamento em B2B são importantes para o desempenho de PMEs. No entanto, a implementação de sistemas CRM nessas empresas pode ser um desafio devido à necessidade de pessoas qualificadas, bem como disponibilidade de tempo e investimento em tecnologias. Assim, sistemas CRM para PMEs não são apenas versões reduzidas das versões em operação em grandes empresas, e sim sistemas específicos que consideram seu contexto e restrições de operação.

A análise da literatura em qualidade do relacionamento e CRM indica que há lacunas de pesquisa em relação ao desenvolvimento, implementação e avaliação desses sistemas considerando o contexto de PMEs e relacionamentos B2B dessas empresas (Casidy \& Nyadzayo, 2017; Harrigan \& Miles, 2014; Wang et al., 2016). Esse estudo vai de encontro à necessidade de pesquisas sobre relacionamentos B2B e o uso de mídias sociais nesses relacionamentos, bem como a consideração do contexto de PMEs nos referidos tópicos (Agnihotri et al., 2016; Bocconcelli et al., 2018; Rodriguez et al., 
2018; Wang et al., 2016). Isto posto, o presente estudo tem como objetivo analisar o processo de comunicação em vendas de uma pequena empresa, considerando os conceitos de CRM Social e qualidade do relacionamento em contextos B2B. A empresa em questão possui sistema CRM, bem como iniciativas de marketing via redes sociais. Assim, as análises e o método para avaliar o sistema CRM social são baseadas na estratégia da empresa para relacionamentos B2B, que busca relacionamentos de longo prazo, além de favorecer a prospecção de novos clientes.

\section{Referencial teórico}

O capítulo é dividido em três seções, sendo a primeira dedicada à identificação dos constructos relacionados à qualidade do relacionamento. A seção seguinte aborda os conceitos sobre CRM e CRM social. Ao final do capítulo são apresentados conceitos relevantes para o entendimento do contexto das PMEs em B2B.

\subsection{Qualidade do relacionamento}

A qualidade do relacionamento $(Q R)$ pode ser definida como a força de um relacionamento e o quanto este atende às necessidades e expectativas das partes (Smith, 1998). O conceito refere-se ao relacionamento entre empresas (business-to-business), entre empresa e o cliente (business-tocustomer), ou entre vendedor e cliente (salesman-to-customer) (Casidy \& Nyadzayo, 2017; Lahiri \& Kedia, 2011). Embora as definições de qualidade do relacionamento variem na literatura, como em termos de agentes envolvidos, seu conceito está relacionado à avaliação geral de atributos do relacionamento (Jiang et al., 2016; Lahiri \& Kedia, 2011). No contexto B2B, o foco da QR é a força e proximidade das relações inter organizacionais e no seu potencial em desenvolver a rede de organizações (Griffith \& Harvey, 2001; Leonidou, 2004).

Não há um consenso sobre a estrutura conceitual de QR, todavia, Jiang et al. (2016) destacou 23 dimensões principais, que incluem a satisfação e confiança, o comprometimento (Almomani, 2019; Rajagopal, 2009; Smith, 1998; Zaefarian et al., 2017), comunicação, cooperação e adaptação (Nyadzayo et al., 2018; Pantouvakis \& Karakasnaki, 2018; Zaefarian et al., 2017). A satisfação refere-se à satisfação geral de um dos agentes, a confiança pode ser medida em referência à honestidade e confiabilidade do canal de suprimento (Rajagopal, 2009). A dimensão comunicação está diretamente relacionada à confiança (Santouridis; Veraki, 2017). Além disso, o compartilhamento frequente de informações de qualidade, informais e presenciais, influencia o comprometimento e a propensão de parceiros B2B permanecerem em um relacionamento (Nyadzayo et al., 2018). O comprometimento ou compromisso relaciona-se à repetição de compra e retenção do comprador, a uma orientação de longo prazo e à expectativa de continuidade do relacionamento (Almomani, 2019; Jiang et al., 2016; Rajagopal, 2009). 
Nesse sentido, o desempenho do relacionamento depende da presença ou ausência dessas dimensões e de como elas se combinam (Zaefarian et al., 2017). Em geral, essas dimensões são avaliadas de acordo com o ponto de vista de uma empresa focal (Jiang et al., 2016). Apesar de estar relacionada à melhoria de desempenho das empresas, como crescimento das vendas e aumento dos lucros, a $Q R$ tem maior ou menor relevância dependendo do contexto em que atuam as empresas (Casidy \& Nyadzayo, 2017; Chatterjee, 2017; de Almeida et al., 2016; Leuthesser, 1997). Em determinados contextos, a evolução do relacionamento em uma parceria comercial é estratégica para os agentes envolvidos, visto que parceiros trabalham por objetivos mútuos, como em grande parte dos relacionamentos B2B (Huntley, 2006).

\subsection{Gestão do relacionamento com o cliente}

O CRM ou Gestão do Relacionamento com o Cliente, termo que surgiu na década de 1990 na área de tecnologia da informação, foi uma resposta à mudança da estratégia de negócios orientada para o produto por uma estratégia focada no cliente (Alshawi et al., 2011; Payne \& Frow, 2005). O CRM pode ser entendido como a implementação prática da teoria de marketing de relacionamento, possibilitando aplicação dessas práticas por meio de tecnologia (Barac et al., 2017). A visão de CRM mais utilizada atualmente é uma abordagem holística e estratégica, com foco em lucratividade, aumento do fluxo de processos de negócios e fidelização dos clientes (Cruz-Jesus et al., 2019; Pedron et al., 2016; Rodriguez et al., 2018; Soltani \& Navimipour, 2016) e depende da coleta extensiva de dados e da distribuição de informações (Fotiadis \& Vassiliadis, 2017).

Os avanços tecnológicos, como as tecnologias Web 2.0 e mídias sociais (blogs, Facebook, LinkedIn), são considerados facilitadores dos sistemas CRM, permitindo que a comunicação deixe de ser apenas unidirecional (Choudhury \& Harrigan, 2014; Cruz-Jesus et al., 2019; Harrigan \& Miles, 2014), aumentando assim a capacidade de armazenar, organizar, processar e analisar dados internos e externos às empresas (Dale Wilson, 2006). Nesse contexto surgiu o conceito de CRM social, que tem como foco o envolvimento do cliente em relacionamentos interativos e bidirecionais (Choudhury \& Harrigan, 2014; Faase et al., 2011).

As mídias sociais devem ser consideradas uma extensão do CRM tradicional da organização (Ancillai et al., 2019; Agnihotri et al., 2017). No sistema CRM social, o envolvimento com o cliente se dá em tempo real e é interativo. O cliente não fica limitado a um papel passivo em seu relacionamento com a empresa. Além da maior disponibilidade de informações sobre produtos da empresa e de concorrentes, os clientes podem expressar suas opiniões para grandes públicos (Malthouse et al., 2013). Assim, o CRM social se baseia na noção do CRM tradicional, mas inclui funções sociais, processos e 
capacidades que consideram a interação entre clientes e empresas, bem como clientes e a sociedade como um todo (Ancillai et al., 2019; Choudhury \& Harrigan, 2014; Kim \& Wang, 2019).

As mídias sociais não exigem conhecimentos técnicos avançados, em sua maioria são gratuitas e não representam complexidade para utilização pelos usuários, sendo favorável para o contexto operacional de PMEs (Atanassova \& Clark, 2015; Harrigan \& Miles, 2014). No entanto, a utilização dessas mídias por PMEs exigem uma abordagem diferente da utilizada em empresas maiores, principalmente devido às restrições de recursos nessas empresas (Cheng \& Shiu, 2019).

As mídias e aplicativos de mídia social fornecem dados para análise de tendências do setor da empresa e lacunas de mercado. Ainda, há maior compartilhamento de conteúdo e conhecimento entre parceiros comerciais, possibilitando a colaboração (Wang et al., 2016; Itani et al. 2017). Além disso, a utilização das mídias sociais é uma maneira econômica de superar barreiras geográficas e de conectarse a diversos grupos que antes estavam fora do alcance dessas PMEs (Atanassova \& Clark, 2015). Assim, sistemas que integram o CRM e as mídias sociais podem ser utilizados como meio de adquirir vantagem competitiva em PMEs, facilitando as relações entre vendedores e clientes (Agnihotri et al., 2017) e diferenciando essas empresas de organizações concorrentes maiores, que têm maior dificuldade em se aproximar do seu cliente (Guha et al., 2018).

\subsection{Pequenas e médias empresas no contexto B2B}

O reconhecimento da contribuição das PMEs para o desenvolvimento econômico nacional, regional e local tem promovido o interesse de pesquisadores das mais diversas áreas, especialmente das ciências sociais aplicadas (Barbosa et al., 2017). Independente do modelo de negócio, geralmente estes empreendimentos possuem recursos restritos (investimento imobilizado e capital de giro) e, de acordo com o seu perfil (faturamento anual e número de funcionários entre outros critérios), são caracterizados como Pequena e Média Empresa (PME) ou mesmo Micro Empresa (ME). No Brasil há diversas classificações para designar PMEs, sendo que nesta pesquisa considerou-se a classificação em relação ao faturamento anual, considerou-se: (i) micro empresa tem receita operacional bruta (ROB) menor ou igual a $\mathrm{R} \$ 360$ mil; (ii) a pequena empresa com ROB maior que $\mathrm{R} \$ 360$ mil e menor ou igual a $R \$ 4,8$ milhões; e a (iii) média empresa tem ROB maior que $R \$ 4,8$ milhões e menor ou igual a $R \$ 300$ milhões (BNDES, 2019; SEBRAE, 2019).

As operações em pequenas e médias empresas são diferentes das grandes organizações, com processos de tomada de decisão menos burocráticos e geralmente centrado no gerente-proprietário (Atanassova \& Clark, 2015). Além disso, a principal restrição para as operações nessas empresas é a escassez de recursos e acesso ao conhecimento, como recursos financeiros (Bocconcelli et al., 2018; Cheng \& Shiu, 2019). 
A capacidade de inovação de PMEs é importante para estabelecer vantagem competitiva em ambientes dinâmicos (Guha et al., 2018). Nesse sentido, restrições relacionadas ao tamanho dessas empresas podem ser superadas por meio de atuação em redes de empresas e colaboração (Buratti et al., 2018; Bocconcelli et al., 2018; Casidy \& Nyadzayo, 2017). Ainda, o networking possibilita ampliar os contatos de clientes e fornecedores para essas empresas, sendo a habilidade de fazer contatos considerada diferencial competitivo para essas empresas (Atanassova \& Clark, 2015). No entanto, apesar dos benefícios dessas estratégias, os conceitos de redes e relacionamentos são pouco explorados em estudos no contexto das PMEs, ainda fortemente associados apenas às habilidades do proprietário-empreendedor ou gerente em termos de networking (Bocconcelli et al., 2018).

O uso de tecnologias para compartilhamento global de informações instituiu novas práticas de marketing em B2B. Acadêmicos destacam o potencial de ferramentas de mídia social para a eficiência e eficácia em processos de trocas em B2B no contexto das PMEs, bem como favorecimento de oportunidades de colaboração (Buratti et al., 2018; Chong et al., 2018; Guha et al., 2018). A utilização de mídias sociais em estratégias de marketing de PMEs complementa o uso de outros sistemas baseados em tecnologia da informação. Embora a utilização desses sistemas gere benefícios em termos de qualidade do relacionamento, há necessidade de recursos organizacionais para desenvolver e manter os mesmos em operação (Cheng \& Shiu, 2019; Chong et al., 2018). Assim, PMEs exigem uma abordagem específica para seu contexto.

\section{Método}

A primeira etapa deste estudo consistiu na revisão da literatura sobre o tema qualidade do relacionamento no contexto $\mathrm{B} 2 \mathrm{~B}$, buscando identificar os constructos relacionados. A revisão também incluiu análise de estudos sobre CRM e CRM social, que é considerado o sistema ideal para o contexto de pequenas e médias empresas em termos de qualidade do relacionamento B2B (Atanassova \& Clark, 2015; Guha et al., 2018; Harrigan \& Miles, 2014). A análise da literatura em qualidade do relacionamento e sistemas CRM indicou lacuna de pesquisa em relação ao desenvolvimento, implementação e avaliação desses sistemas no contexto B2B das PMEs (Alshawi et al., 2011; Casidy \& Nyadzayo, 2017; Harrigan \& Miles, 2014; Wang et al., 2015). Assim, devido à necessidade de maior aprofundamento das análises nesse contexto específico, o método estudo de caso foi utilizado nesta pesquisa.

O método estudo de caso proporciona maior riqueza na coleta de dados e pode ser utilizado para verificar se a teoria proposta está correta ou se um conjunto alternativo de explicações é mais relevante (Miguel, 2012; Yin, 2009). Os procedimentos metodológicos adotados são apresentados na Figura 1, que foi elaborada de acordo com o que é proposto em Dresch et al. (2015), Miguel (2012) e Yin (2009) para condução de pesquisas com o método estudo de caso. 
A segunda etapa, conforme ilustra a Figura 1, corresponde ao planejamento, que envolveu atividades relacionadas à seleção do caso e definição dos meios para coleta de dados, bem como elaboração do protocolo de pesquisa. Essa etapa consiste em planejar como será a terceira etapa, que corresponde à coleta de dados na unidade de análise, com objetivo de extrair grande volume de dados com eficiência. Nesse sentido, a unidade de análise selecionada é uma cervejaria artesanal brasileira de Blumenau, Santa Catarina. Os dados foram coletados no mês de novembro de 2018, por meio de entrevista semiestruturada com o proprietário da empresa, análise documental e observações in loco. A coleta de dados considerou múltiplas fontes conforme sugerido em Dresch et al. (2015). Ainda, elaborou-se roteiro para análise dos dados.

Figura 1

Procedimentos Metodológicos

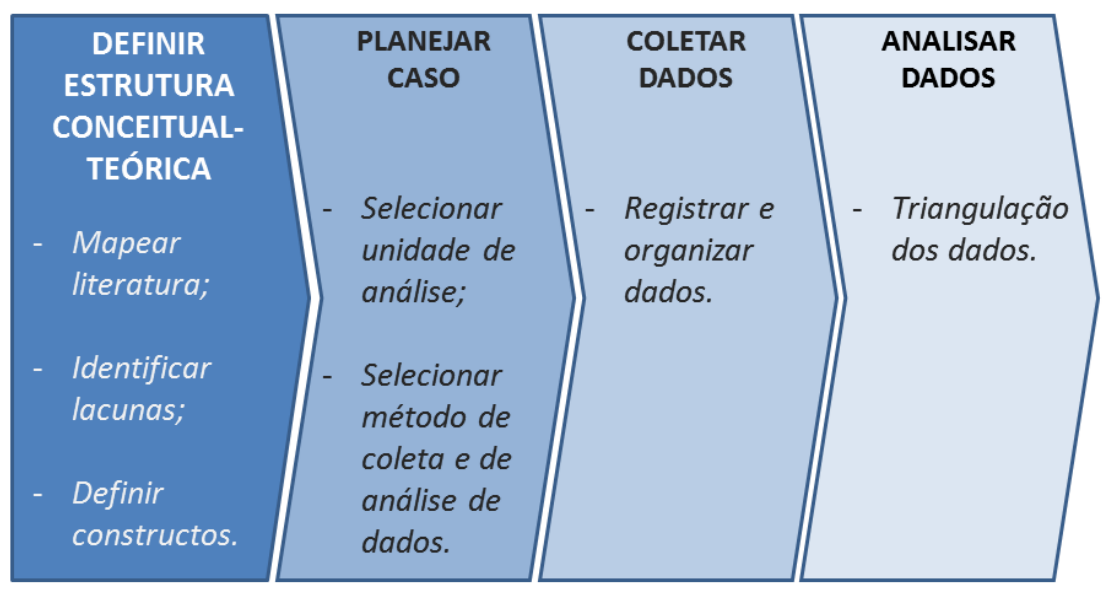

Fonte: Elaborado pelos autores (2019).

A quarta etapa consistiu na análise dos dados coletados. Segundo Eisenhardt (1989), o uso de múltiplas fontes de dados e a iteração com os constructos desenvolvidos a partir da literatura possibilitam que o pesquisador alcance maior validade construtiva da pesquisa. O roteiro da quarta e última etapa do estudo é composta pelas seguintes atividades: (i) descrição da situação de estudo por meio de entrevista semiestruturada com o gerente/proprietário da empresa; (ii) caracterização do processo de comunicação para o contexto de estudo com base na literatura; (iii) detalhamento de método quantitativo para avaliação do processo de comunicação da empresa, considerando os construtos identificados na primeira etapa da pesquisa.

\section{Apresentação e análise dos resultados}

Este capítulo está subdividido em quatro seções. A primeira e segunda seções apresentam a unidade de análise e caracterização de seu processo de vendas. A seção 4.3 descreve as melhorias 
adotadas no processo de comunicação da empresa. Na seção 4.4 são apresentados os indicadores utilizados para avaliação e sua implicação teórica.

\subsection{Apresentação da unidade de análise}

O presente estudo foi realizado em uma cervejaria artesanal localizada na cidade de Blumenau, SC. A cervejaria iniciou as operações no ano de 2014, atuando exclusivamente no modelo de negócios de contract brewing, ou seja, toda sua produção é terceirizada. Mesmo sendo uma empresa jovem, a mesma já acumula premiações de âmbito nacional com destaque para as premiações obtidas no Festival Brasileiro da Cerveja, além de menções em revistas especializadas do meio.

Atualmente a empresa conta com 4 funcionários e seu faturamento médio é de 60 mil reais mensais. Suas atividades se concentram na área de marketing, vendas e distribuição, além das atividades administrativas inerentes a qualquer tipo de empreendimento. Com relação ao marketing, a empresa foca as ações em mídias sociais utilizando-se de canais como Facebook, Instagram e WhatsApp. Com relação a atividade de vendas, a empresa possui um processo heterogêneo contando com vendedor interno, vendedor externo e representantes. A empresa conta com 226 pontos de venda com relacionamento, sendo que destes, 137 são clientes regulares. O foco principal das vendas é a região catarinense do Vale do Itajaí, onde, um vendedor externo dedica seu tempo para visitar os pontos de venda atuais, além de prospectar novos pontos. Em segundo plano, existe uma atuação mais ampla, considerando o estado de SC, onde algumas cidades estratégicas como Joinville, Chapecó, Florianópolis e Criciúma são atendidas. As vendas nestas cidades são realizadas através de representantes comerciais. As visitas aos clientes são realizadas pelo vendedor e representantes mediante agendamento.

Adicionalmente, existe um trabalho de venda interna realizado por um atendente, que é responsável por entrar em contato com todos os clientes, agendar visitas e prospectar novos pontos de venda. O contato geralmente é realizado via ligações telefônicas ou mídias sociais, sendo que o último está cada vez mais popular e é a principal forma de contato com os clientes. Atualmente $90 \%$ das vendas são realizadas para outras empresas (atacadistas ou varejistas) caracterizando uma relação B2B, sendo que estas empresas também são classificadas como PMEs ou MEs. Com relação à distribuição, a empresa opera com sistema de entrega próprio para a região do Vale do Itajaí e com empresas parceiras (transportadoras e/ou serviços de entrega rápida) que atendem as demais regiões e eventualmente realizam entregas emergenciais.

Todas as interações com clientes são registradas no sistema CRM utilizado pela empresa. De acordo com o proprietário, a utilização do CRM é vista como estratégica e é imperativo que as informações estejam atualizadas, o que ocasiona certa dificuldade em algumas situações.

O proprietário elencou as problemáticas no processo de comunicação com os clientes. Em primeiro lugar, o transtorno do gerenciamento de vários perfis de redes sociais além de vários usuários 
com acesso ao CRM. Em segundo lugar, as informações muitas vezes estão dispersas. Por exemplo, é muito comum uma negociação de venda iniciar via ligação telefônica e ser concluída via Facebook ou WhatsApp. Por fim, cada cliente geralmente usa uma mídia com mais frequência e é complicado enviar informações personalizadas para cada cliente quando o sistema não é integrado.

\subsection{Caracterização do processo de comunicação em vendas na empresa}

No contexto deste estudo, com base na coleta de dados, constatou-se que o foco no cliente toma como base o varejista, ou seja, bares, restaurantes, empórios ou casas especializadas na venda de cervejas artesanais. Por se tratar de um contexto B2B, verificou-se que a empresa foca nos elementos do seu relacionamento com compradores, bem como nos elementos dessa relação que vão influenciar as interações entre varejista e o consumidor final da cervejaria. Sendo assim, a qualidade do relacionamento entre a cervejaria e o varejista é importante na criação de valor, volume de vendas e para o seu desempenho financeiro (Leonidou, 2004; Grewal et al., 2017; Casidy \& Nyadzayo, 2017).

No contexto B2B, o CRM social auxilia na QR e fortalece o capital social da empresa ao combinar as funcionalidades do CRM e de mídias sociais (Rodriguez et al., 2018). Nesse sentido, as mídias sociais inseriram propriedades interativas e relacionais. Os clientes da cervejaria geralmente utilizam as mídias sociais para comparar produtos, pesquisa de mercado e no relacionamento com vendedores. Os vendedores utilizam as mídias sociais em todas as etapas da venda, desde a prospecção até o pós-venda. As informações em mídias sociais permitem que vendedores adaptem suas ofertas e a apresentação das mesmas para atender à demanda dos clientes.

Itani et al. (2017) enfatiza que as empresas utilizam mídias sociais para gerar conteúdo, como por meio de blogs, e desenvolver redes, como redes sociais ou comunidades online, para prospecção e interação com clientes. A utilização de redes sociais tende a aumentar a performance de negócios de diversas maneiras. Considerando o foco em marketing e vendas, há maior exposição da marca e produtos (Wang et al., 2016; Dekoulou \& Trivellas, 2017). Além disso, as mídias sociais são importantes para o processo de inovação em uma empresa e facilitadoras para comunicação de PMEs com seus clientes (Wamba \& Carter, 2014; Wang et al., 2016).

O CRM social é considerado a tecnologia mais apropriada para o contexto das PMEs (Atanassova \& Clark, 2015; Guha et al., 2018; Harrigan \& Miles, 2014). De acordo com os dados coletados na entrevista e a análise da literatura, o uso integrado de mídias sociais e sistemas CRM fornece uma combinação ideal em termos de QR e desempenho dos negócios em PMEs. Possibilita maior visibilidade da empresa e o contato com consumidores e clientes, além de melhorar o desempenho da comunicação com fornecedores e identificação de possíveis parceiros no canal de distribuição (Wang et al., 2016). 


\subsection{Conectando CRM social e QR}

Este estudo avalia o resultado da utilização de mídias sociais no contexto B2B. A utilização de mídias sociais dá suporte a diferentes passos no processo de vendas (Ancillai et al., 2019; Andzulis et al., 2012; Moncrief et al., 2015; Itani et al., 2017). As análises do estudo são baseadas nas seguintes premissas: (i) o comprador tem conhecimento a respeito do produto, que neste caso é a cerveja artesanal; (ii) o comprador virá ou não a adquirir o produto; (iii) o varejista pode ser comprador ou iniciar esse processo de compra pela primeira vez; e (iv) esse cliente busca um produto de qualidade e precisa de conveniência no atendimento e reposição de estoque.

A literatura enfatiza a importância da comunicação entre comprador e vendedor para fortalecer o relacionamento no processo de troca. Em B2B, a comunicação durante o processo de venda pode ser a razão de conquistar o cliente, bem como da satisfação do cliente (Agnihotri et al., 2016; Itani et al.,2017; Chatterjee 2017). Outro ponto relevante no processo de venda em B2B é a capacidade de resposta, que deve ser considerada em termos de agilidade e conteúdo (Agnihotri et al., 2016) e adaptação de comportamento em relação à necessidade do cliente (Itani et al., 2017). A capacidade de resposta do vendedor relaciona-se com as dimensões de comunicação e satisfação social da QR (Huntley, 2006; Jiang et al., 2016). No contexto do estudo, a empresa preza por construir relacionamentos duradouros com seus clientes, o que justifica os investimentos em sistemas como o CRM social (Faase et al., 2011; Jiang et al., 2016).

O processo de vendas pode ter um ciclo longo, pode ser um processo complexo e até mesmo envolver um maior número de tomadores de decisão, que são fatores que se alteram conforme o contexto de cada empresa (Rodriguez et al., 2018). Assim, a comunicação no processo de venda da empresa deste estudo foi analisada para identificar as principais atividades em que há contato direto com o varejista: apresentação dos produtos e serviços utilizando uma descrição objetiva e imagens ilustrativas, agendamento de visitas, negociação (preços, frete, prazos de entrega e prazos para pagamento) e comunicação em geral.

A empresa do estudo é pequena e possui restrições em termos de recursos financeiros, recursos humanos e infraestrutura. As atividades descritas acima são rotineiras e ocorrem por meio dos seguintes canais: presencial, chamada telefônica e online, com suporte de sites ou aplicativos de redes sociais. Além disso, é comum um processo de vendas exigir a interação por meio de mais de um canal até que seja encerrado.

Assim, considerando a tendência das tecnologias digitais nas operações das empresas, bem como o próprio perfil da empresa, a mesma optou por focar em um processo de comunicação baseado nos sistemas CRM social (Alshawi et al., 2011; Atanassova \& Clark, 2015; Guha et al., 2018; Harrigan \& 
Miles, 2014). Basicamente, toda a comunicação com o varejista é suportada pela integração entre aplicativos e sites de redes sociais, o sistema CRM utilizado pela empresa e um sistema de chat online.

Do ponto de vista do varejista (cliente), o sistema integrado utilizado pela empresa agrega valor, possibilitando realizar várias atividades corriqueiras (por exemplo, questionamentos ao vendedor antes de concretizar a compra, recebimento de informações relacionadas ao produto, além de sugestões de novas compras baseadas no seu perfil) por meio da interface de aplicativos e sites de redes sociais amplamente conhecidos. O comprador consulta as formas e prazo para pagamento, opção de frete, entre outras informações pertinentes para concluir a compra. As respostas não são automáticas. Ou seja, o comprador percebe que existe outra pessoa ali dedicando seu tempo para realizar o atendimento e sanar todas as possíveis dúvidas, o que transmite segurança no atendimento.

O CRM social possibilita uma interação individual, onde o cliente sente como se recebesse um tratamento exclusivo (Faase et al., 2011). Além disso, traz mais simplicidade e agilidade ao processo, porque o pedido e a conversa com o vendedor ficam organizados em uma linha do tempo fácil de ser consultada. Essa comunicação abrange todo o processo de compra (Agnihotri et al., 2016). Desta forma, realizar uma compra pelo celular ou computador em qualquer lugar é tão fácil quanto usar um aplicativo de mensagens.

Um dos aspectos mais importantes durante o processo de vendas é a capacidade do vendedor em comunicar a informação, visto que os clientes não ficarão satisfeitos com a interação se a informação não for comunicada com precisão ou clareza (Agnihotri et al., 2016). A satisfação com a comunicação e o serviço prestado aos varejistas no processo de venda diferenciam a empresa do estudo de seus concorrentes menos coordenados (Griffith \& Harvey, 2001; Lahiri \& Kedia, 2011), evidenciando a utilização de inteligência competitiva e a orientação ao aprendizado pelos vendedores (Itani et al., 2017). Assim, com base nas compras realizadas, periodicamente é solicitado ao cliente que avalie o atendimento, considerando os mecanismos de pesquisa online, a exemplo dos indicadores Net Promoter Score (NPS) com o objetivo de avaliar o nível de qualidade do serviço e a capacidade de resolução dos problemas (Casidy \& Nyadzayo, 2017; Dekoulou,\& Trivellas, 2017). E não existe melhor momento para que o fornecedor demonstre comprometimento com o cliente do que quando os problemas aparecem, seja qual for a sua origem (Bettencourt et al., 2015).

Do ponto de vista da empresa (ou fornecedor), o sistema de atendimento via chat online, integrado com aplicativos e sites de redes sociais, significa mais praticidade, e elimina a necessidade de ficar conectado em várias plataformas ao mesmo tempo. Considerando as restrições de recursos em PMEs, é vantajoso que seja uma proposta simplificada nesse sentido (Atanassova \& Clark, 2015; Wang et al., 2016).

O sistema integrado permite ao atendente consultar o histórico de mensagens e dar andamento ao processo de venda. A interface de chat possibilita o envio de fotos e detalhes sobre os produtos para 
que o comprador possa fechar o seu pedido. Mesmo quando o atendente não está online os contatos são registrados na linha do tempo.

Ao registrar a conversa com o cliente, o sistema de chat online identifica cada etapa e sugere quais ações precisam ser tomadas para dar andamento ao pedido. Informações comuns sobre o pedido, como o aviso sobre a expedição do produto ou pagamento recebido, são realizadas de forma automática sem intervenção do atendente. Cada contato realizado é registrado no sistema de CRM.

O sistema possibilita agendamento de visitas, considerando que as agendas dos vendedores e representantes são compartilhadas. Dessa forma, além do registro do contato outras informações para tomada de decisões estratégicas são armazenadas a exemplo de quais produtos foram mencionados durante a conversa, se foi finalizado o pedido e com qual opção de pagamento/frete e a data da última visita.

Em resumo, as principais características do sistema integrado de chat online são:

- Agilidade e simplicidade da utilização de aplicativos de mensagens;

- Velocidade no contato entre comprador e vendedor para sanar eventuais dúvidas através da identificação do interesse na compra;

- Visualização dos dados do contato do cliente em um único lugar;

- Compartilhamento de agenda para os vendedores e representantes, simplificando o processo de agendamento dos compromissos;

- Praticidade ao dar andamento e finalizar o processo de vendas sem a necessidade de alternar entre vários sistemas.

Segundo Bettencourt et al. (2015), iniciativas como esta chamam a atenção para a prioridade que um fornecedor coloca ao atendimento das necessidades do comprador. Os fornecedores que se destacam em iniciativas de relacionamento enxergam os clientes como colaboradores buscando objetivos mutuamente benéficos e não como adversários com agendas conflitantes.

\subsection{Avaliação e discussão}

A utilização de mídias sociais tem assumido um papel protagonista, considerando todos os tipos de comunicação e está criando um potencial único para melhoria de performance, tanto individual como organizacional (Itani et al., 2017). Nesse sentido, para avaliar a utilização do CRM social no processo de vendas, a empresa analisa sua performance periodicamente. Tais avaliações podem revelar demandas de serviço que não estão sendo totalmente atendidas possibilitando ajustes futuros (Payne \& Frow, 2005). Daí decorre a necessidade de que sejam disponibilizadas informações adequadas (e recorrentes) para a viabilização das avaliações (Paladini, 2011). 
Nesse sentido, a construção de indicadores é fundamental para avaliar o desempenho dos processos realizados conforme exposto na seção 4.3. A empresa já possui sistema de avaliação da qualidade com base no sistema CRM. No entanto, considerando a literatura, outros indicadores devem compor a avaliação. Assim, o Quadro 1 detalha os indicadores propostos para a empresa.

\section{Quadro 1}

Indicadores de Desempenho

\begin{tabular}{|c|c|c|c|c|}
\hline Indicador & Objetivo & Justificativa & Referência & Métricas utilizadas \\
\hline 1 & $\begin{array}{l}\text { Determinar } \\
\text { a satisfação } \\
\text { do cliente }\end{array}$ & $\begin{array}{l}\text { Avaliar o } \\
\text { desempenho no } \\
\text { relacionamento } \\
\text { com o cliente }\end{array}$ & $\begin{array}{l}\text { Kim \& Wang, 2019; } \\
\text { Chatterjee (2017); Dekoulou } \\
\text { \& Trivellas (2017); Soltani \& } \\
\text { Navimipour (2016); Wang et } \\
\text { al. (2016); Choudhury \& } \\
\text { Harrigan (2014); Rodriguez } \\
\text { et al. (2012); Alshawi et al. } \\
\text { (2011); }\end{array}$ & $\begin{array}{l}\text { Percentual mensal de } \\
\text { atendimentos } \\
\text { avaliados como } \\
\text { Contente ou Muito } \\
\text { contente. Mínimo de } \\
80 \% \text {. }\end{array}$ \\
\hline 2 & $\begin{array}{l}\text { Estimar a } \\
\text { performance } \\
\text { de vendas }\end{array}$ & $\begin{array}{l}\text { Ajustar a oferta em } \\
\text { relação à demanda }\end{array}$ & $\begin{array}{l}\text { Kim \& Wang, 2019; } \\
\text { Chatterjee (2017); Dekoulou } \\
\text { \& Trivellas (2017); Fotiadis \& } \\
\text { Vassiliadis (2017); Rodriguez } \\
\text { et al. (2012); Alshawi et al. } \\
\text { (2011); }\end{array}$ & $\begin{array}{l}\text { Percentual de vendas } \\
\text { por produto por } \\
\text { trimestre. Variação } \\
\text { tolerável de } 10 \% \text {. }\end{array}$ \\
\hline 3 & $\begin{array}{l}\text { Escala de } \\
\text { utilização de } \\
\text { mídias } \\
\text { sociais }\end{array}$ & $\begin{array}{l}\text { Avaliar quais } \\
\text { recursos providos } \\
\text { pelas mídias sociais } \\
\text { são utilizados }\end{array}$ & $\begin{array}{l}\text { Itani et al. (2017); Wang et } \\
\text { al. (2016); Rodriguez et al. } \\
\text { (2012) }\end{array}$ & $\begin{array}{l}\text { Percentual de } \\
\text { contatos de vendas } \\
\text { realizados por mídias } \\
\text { sociais. Mínimo de } \\
50 \% \text {. }\end{array}$ \\
\hline 4 & $\begin{array}{l}\text { Escala de } \\
\text { utilidade e } \\
\text { eficiência } \\
\text { das mídias } \\
\text { sociais }\end{array}$ & $\begin{array}{l}\text { Identificar o grau } \\
\text { de fechamento de } \\
\text { vendas quando as } \\
\text { mídias sociais são } \\
\text { utilizadas }\end{array}$ & $\begin{array}{l}\text { Kim \& Wang, 2019; } \\
\text { Itani et al. (2017); Pedron et } \\
\text { al. (2017); Soltani \& } \\
\text { Navimipour (2016); Wang et } \\
\text { al. (2016); Rodriguez et al. } \\
\text { (2012); Alshawi et al. (2011) }\end{array}$ & $\begin{array}{l}\text { Percentual de vendas } \\
\text { por mês. Mínimo de } \\
30 \% \text {. }\end{array}$ \\
\hline
\end{tabular}

Fonte: Elaborado pelos autores. 
A empresa avaliou os atendimentos realizados no período de outubro a novembro de 2018, totalizando 61 clientes atendidos. Para avaliação do indicador 1, foram enviadas perguntas para que o cliente respondesse ao finalizar um atendimento. Destes 42 responderam, equivalente a $68 \%$ de adesão, sendo que $83 \%$ dos respondentes relataram estar contentes ou muito contentes com o atendimento realizado.

Os indicadores 2 e 4 foram avaliados a partir dos contatos realizados e o fechamento das vendas, indicando um aumento de $11 \%$ no número de fechamentos em relação ao mesmo período no ano anterior. No contexto da escala de utilidade, $71 \%$ considera que as mídias sociais facilitam o trabalho.

E finalmente, o indicador 3 foi avaliado diretamente com os vendedores. Considerando a escala de utilização de mídias sociais, $85 \%$ afirmam usar os recursos das mídias sociais da melhor maneira para facilitar o trabalho sendo que $61 \%$ dos contatos realizados via mídias sociais foram respondidos de forma a encaminhar para o processo de fechamento de vendas.

Assim, ao analisar os dados coletados, observou-se que a utilização de CRM Social no contexto de vendas possibilita a geração de resultados positivos em relacionamentos B2B, permitindo que os vendedores aumentem seu desempenho e que possam construir relacionamentos com os clientes de forma a tornar futuras transações mais assertivas, especialmente no contexto da oferta de produtos e serviços.

\section{Considerações finais}

Pesquisas acadêmicas reconhecem que as mídias sociais estão se tornando uma ferramenta dominante no processo de vendas, afetando o ambiente de vendas em sua totalidade. O presente trabalho objetivou apresentar o processo de comunicação em vendas no contexto das PMEs baseado no conceito de $Q R$, em mídias sociais e sistema CRM. A empresa objeto do estudo é uma cervejaria que utiliza um sistema do tipo CRM social para aumento da confiança no processo de comunicação e da sua capacidade de resposta, além da satisfação do cliente em termos de atendimento individualizado. Adicionalmente propôs-se a utilização de indicadores para avaliar o processo, que consideraram a performance em vendas, nível de atendimento, escala de utilização e efetividade na utilização das mídias sociais. Na prática, o estudo comprova que a adoção de sistemas do tipo CRM Social é importante para a empresa do estudo, devido ao interesse na construção de relacionamentos de longo prazo, bem como para a prospecção de novos clientes.

Este estudo também contribuiu para a teoria de qualidade do relacionamento B2B ao analisar a adoção de sistemas CRM baseados em mídias sociais em PMEs, bem como identificar e aplicar indicadores para avaliação desses sistemas. Destaca-se, como limitação deste estudo, a análise de caso único, considerando apenas a visão da empresa. 
Estudos futuros podem abranger outras empresas no contexto de PMEs identificando como o CRM Social é utilizado, sua aceitação, oportunidades e desafios. Ainda, estudo futuros podem analisar a utilização de sistemas do tipo CRM Social sob o ponto de vista do cliente.

\section{Referências}

Agnihotri, R., Dingus, R., Hu, M. Y., \& Krush, M. T. (2016). Social media: Influencing customer satisfaction in B2B sales. Industrial Marketing Management, 53, 172-180.

Agnihotri, R., Trainor, K. J., Itani, O. S., \& Rodriguez, M. (2017). Examining the role of sales-based CRM technology and social media use on post-sale service behaviors in India. Journal of Business Research, 81, 144-154.

Almomani, H. Q. (2019). Relationship Quality as Predictor of B2B Customer Loyalty in the Pharmaceutical Sector: Evidence from Jordan. Journal of Relationship Marketing, 1-16.

Alshawi, S., Missi, F., \& Irani, Z. (2011). Organisational, technical and data quality factors in CRM adoption-SMEs perspective. Industrial Marketing Management, 40(3), 376-383.

Ancillai, C., Terho, H., Cardinali, S., \& Pascucci, F. (2019). Advancing social media driven sales research: Establishing conceptual foundations for B-to-B social selling. Industrial Marketing Management.

Andzulis, J. M., Panagopoulos, N. G., \& Rapp, A. (2012). A review of social media and implications for the sales process. Journal of Personal Selling \& Sales Management, 32(3), 305-316.

Atanassova, I., \& Clark, L. (2015). Social media practices in SME marketing activities: A theoretical framework and research agenda. Journal of customer behaviour, 14(2), 163-183.

Barac, D., Ratkovic-Živanovic, V., Labus, M., Milinovic, S., \& Labus, A. (2017). Fostering partner relationship management in $\mathrm{B} 2 \mathrm{~B}$ ecosystems of electronic media. Journal of Business \& Industrial Marketing, 32(8), 1203-1216.

Barbosa de Moraes, M., Armstrong Santos de Miranda, M., de Araujo Querido Oliveira, E. A., \& Marques dos Santos, E. (2017). Cultura de inovação em micro e pequenas empresas dos setores aeronáutico e de tecnologia da informação. Exacta, 15(3).

Bettencourt, L. A., Blocker, C. P., Houston, M. B., \& Flint, D. J. (2015). Rethinking customer relationships. Business Horizons, 58(1), 99-108.

BNDES. (2019). Guia do Financiamento. [página Web]. Disponível em: <https://www.bndes.gov.br/wps/portal/site/home/financiamento/guia/quem-pode-sercliente/>. Acesso em 26 de junho de 2019.

Buratti, N., Parola, F., \& Satta, G. (2018). Insights on the adoption of social media marketing in B2B services. The TQM Journal, 30(5), 490-529.

Casidy, R., \& Nyadzayo, M. (2017). Drivers and outcomes of relationship quality with professional service firms: An SME owner-manager perspective. Industrial Marketing Management. 
Chang, H. H., Wong, K. H., \& Fang, P. W. (2014). The effects of customer relationship management relational information processes on customer-based performance. Decision Support Systems, 66, 146-159.

Chatterjee, S. C. (2017). B2B relationship calculus: Quantifying resource effects in service-dominant logic. Journal of the Academy of Marketing Science, 45(3), 402-427.

Cheng, C. C., \& Shiu, E. C. (2019). How to enhance SMEs customer involvement using social media: The role of Social CRM. International Small Business Journal, 37(1), 22-42.

Chong, W. K., Man, K. L., \& Kim, M. (2018). The impact of e-marketing orientation on performance in Asian SMEs: a B2B perspective. Enterprise Information Systems, 12(1), 4-18.

Choudhury, M. M., \& Harrigan, P. (2014). CRM to social CRM: the integration of new technologies into customer relationship management. Journal of Strategic Marketing, 22(2), 149-176.

Cruz-Jesus, F., Pinheiro, A., \& Oliveira, T. (2019). Understanding CRM adoption stages: empirical analysis building on the TOE framework. Computers in Industry, 109, 1-13.

de Almeida Vilela, B., de Souza Alves, C., Correia Ferreira, R. F., Alves de Freitas, K., \& Canedo de Souza Junior, W. (2016). Coordenação em cadeias de suprimentos: o papel da tecnologia da informação e da gestão orientada por processos. Exacta, 14(4).

Dale Wilson, R. (2006). Developing new business strategies in B2B markets by combining CRM concepts and online databases. Competitiveness Review: An International Business Journal, 16(1), 38-43.

Dekoulou, P., \& Trivellas, P. (2017). Organizational structure, innovation performance and customer relationship value in the Greek advertising and media industry. Journal of Business \& Industrial Marketing, 32(3), 385-397.

Demo, G., Fogaca, N., Ponte, V., Fernandes, T., \& Cardoso, H. (2015). Customer Relationship Management (CRM): State of the Art, Bibliometric Review of High-Quality Brazilian Production, Institutionalization of Research in Brazil and Research Agenda. RAM. Revista de Administração Mackenzie, 16(5), 127-160.

Dresch, A., Lacerda, D. P., \& Miguel, P. A. C. (2015). A distinctive analysis of case study, action research and design science research. Revista brasileira de gestão de negócios, 17(56), 1116-1133.

Eisenhardt, K. M. (1989). Building theories from case study research. Academy of management review, v. 14 , n. 4, p. 532-550.

Faase, R., Helms, R., \& Spruit, M. (2011). Web 2.0 in the CRM domain: defining social CRM. International Journal of Electronic Customer Relationship Management, 5(1), 1-22.

Fotiadis, Anestis K., and Chris Vassiliadis. (2017). Being customer-centric through CRM metrics in the B2B market: the case of maritime shipping. Journal of Business \& Industrial Marketing 32.3: 347-356.

Grewal, D., Levy, M., \& Kumar, V. (2009). Customer experience management in retailing: An organizing framework. Journal of Retailing, 85(1), 1-14. 
Grewal, D., Roggeveen, A. L., Sisodia, R., \& Nordfält, J. (2017). Enhancing customer engagement through consciousness. Journal of Retailing, 93(1), 55-64.

Griffith, D. A., \& Harvey, M. G. (2001). An intercultural communication model for use in global interorganizational networks. Journal of International Marketing, 9(3), 87-103.

Guha, S., Harrigan, P., \& Soutar, G. (2018). Linking social media to customer relationship management (CRM): a qualitative study on SMEs. Journal of Small Business \& Entrepreneurship, 30(3), 193214.

Harrigan, P., \& Miles, M. (2014). From e-CRM to s-CRM. Critical factors underpinning the social CRM activities of SMEs. Small Enterprise Research, 21(1), 99-116.

Huntley, J. K. (2006). Conceptualization and measurement of relationship quality: Linking relationship quality to actual sales and recommendation intention. Industrial Marketing Management, 35(6), 703-714.

Itani, O. S., Agnihotri, R., \& Dingus, R. (2017). Social media use in B2B sales and its impact on competitive intelligence collection and adaptive selling: Examining the role of learning orientation as an enabler. Industrial Marketing Management, 66, 64-79.

Jiang, Z., Shiu, E., Henneberg, S., \& Naude, P. (2016). Relationship quality in business to business relationships-Reviewing the current literatures and proposing a new measurement model. Psychology \& Marketing, 33(4), 297-313.

Kim, H. G., \& Wang, Z. (2019). Defining and measuring social customer-relationship management (CRM) capabilities. Journal of Marketing Analytics, 7(1), 40-50.

Lahiri, S., \& Kedia, B. L. (2011). Determining quality of business-to-business relationships: A study of Indian IT-enabled service providers. European Management Journal, 29(1), 11-24.

Leonidou, L. C. (2004). Industrial manufacturer-customer relationships: The discriminating role of the buying situation. Industrial Marketing Management, 33(8), 731-742.

Leuthesser, L. (1997). Supplier relational behavior: An empirical assessment. Industrial marketing management, 26(3), 245-254.

Malthouse, E. C., Haenlein, M., Skiera, B., Wege, E., \& Zhang, M. (2013). Managing customer relationships in the social media era: Introducing the social CRM house. Journal of Interactive Marketing, 27(4), 270-280.

Miguel, P. A. C. (2012). Metodologia de pesquisa em engenharia de produção e gestão de operações. Elsevier.

Moncrief, W. C., Marshall, G. W., \& Rudd, J. M. (2015). Social media and related technology: Drivers of change in managing the contemporary sales force. Business Horizons, 58(1), 45-55.

Nyadzayo, M. W., Matanda, M. J., \& Rajaguru, R. (2018). The determinants of franchise brand loyalty in B2B markets: An emerging market perspective. Journal of Business Research, 86, 435-445.

Paladini, E. P. (2011) Avaliação Estratégica Da Qualidade. 2 ed. São Paulo: Atlas SA. 
Pantouvakis, A.; Karakasnaki, M. (2018). The role of agility and organisational learning culture in forming long lasting relations in shipping. International Journal of Shipping and Transport Logistics, v. 10, n. 2, p. 160-180.

Payne, A., \& Frow, P. (2005). A strategic framework for customer relationship management. Journal of marketing, 69(4), 167-176.

Pedron, C. D., W. N. Picoto, G. Dhillon, \& M. Caldeira. (2016). Value-focused objectives for CRM system adoption. Industrial Management and Data Systems 116 (3):526-45.

Rajagopal, A. (2009). Buyer-supplier relationship and operational dynamics. Journal of the Operational Research Society, 60(3), 313-320.

Rodriguez, M., Peterson, R. M., \& Krishnan, V. (2012). Social media's influence on business-to-business sales performance. Journal of Personal Selling \& Sales Management, 32(3), 365-378.

Rodriguez, M., Peterson, R. M., \& Krishnan, V. (2018). Impact of CRM technology on sales process behaviors: empirical results from US, Europe, and Asia. Journal of Business-to-Business Marketing, 25(1), 1-10.

Santouridis, I., \& Veraki, A. (2017). Customer relationship management and customer satisfaction: the mediating role of relationship quality. Total Quality Management \& Business Excellence, 28(910), 1122-1133.

SEBRAE - Serviço Brasileiro de Apoio às Micro e Pequenas Empresas. (2019). Lei Geral das micro e pequenas empresas. Disponível em:

$<$ http://www.sebrae.com.br/sites/PortalSebrae/artigos/entenda-as-diferencas-entremicroempresa-pequena-empresa-e mei,03f5438af1c92410VgnVCM100000b272010aRCRD>. Acesso em: 26 de junho de 2019.

Smith, J. B. (1998). Buyer-seller relationships: similarity, relationship management, and quality. Psychology \& Marketing, 15(1), 3-21.

Soltani, Z., \& Navimipour, N. J. (2016). Customer relationship management mechanisms: a systematic review of the state of the art literature and recommendations for future research. Computers in Human Behavior, 61, 667-688.

Wamba, S. F., \& Carter, L. (2016). Social media tools adoption and use by SMEs: An empirical study. In Social Media and Networking: Concepts, Methodologies, Tools, and Applications (pp. 791806). IGI Global.

Wang, W. Y., Pauleen, D. J., \& Zhang, T. (2016). How social media applications affect B2B communication and improve business performance in SMEs. Industrial Marketing Management, 54, 4-14.

Yin, Robert K. (2009). Case study research: Design and methods (applied social research methods). London and Singapore: Sage.

Zaefarian, G.; Thiesbrummel, C.; Henneberg, S. C.; Naudé P. (2017) Different recipes for success in business relationships. Industrial Marketing Management, v. 63, p. 69-81. 